\title{
Effects of environmental novelty on filial behavior in imprinted ducklings
}

\author{
LEONARD A. EISERER \\ Elizabethtown College, Elizabethtown, Pennsylvania
}

\begin{abstract}
Environmental novelty was manipulated in imprinted ducklings by varying the illumination level of the experimental apparatus. Bright illumination disrupted filial behavior in subjects that had received little prior exposure to the imprinting object, with approach being suppressed more when the object was stationary than when it was moving. In subjects with prolonged prior imprinting exposure, bright illumination enhanced filial behavior. These effects can be understood if one assumes that environmental novelty has an energization effect while also eliciting responses that are incompatible with filial behavior.
\end{abstract}

Stimuli that induce distress calls or flight responses in young precocial birds can often facilitate the attachment that those birds display towards an imprinting object. Thus, environmental novelty, shock, loud noise, conditioned fear stimuli, low temperature, and physical abuse have all been found to enhance filial behavior relative to control conditions lacking maltreatment (Candland, Nagy, \& Conklyn, 1963; Fischer, 1970; Hess, 1959; Moltz, Rosenblum, \& Halikas, 1959; Pitz \& Ross, 1961; Salzen, 1970). This facilitation makes sense when one views the imprinting object as a comforting stimulus that alleviates distress (Moltz, 1960; Salzen, 1967). Thus, distress could be expected to "drive" a bird towards the comforting imprinting object (Eiserer, 1978; Sluckin, 1972).

Sometimes, however, facilitation effects have not occurred under conditions in which they might have been expected. Brown and Hamilton (1977) tested the response of chicks to a stationary imprinting object in an environment that differed from the birds' rearing environment and found that approach to the imprinting object declined in the novel environment. Brown and Hamilton concluded that "with sufficiently intense fear, incompatible responses (e.g., immobility) will lower approach" (p. 791).

In related work, Sluckin, Berryman, Mayes, and Mann (1979) tested the hypothesis that "more powerful" imprinting could resist disruption by environmental novelty. Specifically, they employed a moving as well as a stationary imprinting object, on the assumption that subjects imprint more strongly to a moving object. In support of Sluckin et al.'s hypothesis, novelty in the testing environment disrupted approach to the stationary, but not to the moving, object.

Reprint requests should be sent to Leonard A. Eiserer, Psychology Department, Elizabethtown College, Elizabethtown, Pennsylvania 17022 .
The present work further examined the relationship between strength of imprinting and the subsequent susceptibility of filial behavior to disruption or facilitation by environmental novelty. In doing so, the work varied duration of initial exposure to the imprinting object in order to provide a somewhat more direct manipulation of "strength of imprinting" than was used by Sluckin et al. (1979).

A second objective of the present research was to replicate and extend the finding by Sluckin et al. (1979) that approach to a stationary imprinting object is more easily disrupted than approach to a moving object. In the Sluckin et al. experiment, attachment to the stationary object was accomplished through prolonged exposure to that stationary object. In the present study, the subjects were exposed to a moving object to the point at which that same object would elicit approach even if it remained stationary during its presentation (Hoffman, Eiserer, \& Singer, 1972). The present work, then, sought to determine whether Sluckin et al.'s finding would still occur when the stationary stimulus had acquired behavioral control through a different exposure procedure.

\section{EXPERIMENT 1}

\begin{abstract}
Method
Subjects. Eighteen Khaki Campbell ducklings (Anas platyrhynchos) were hatched, in visual isolation, from eggs obtained from Willow Farms, Richland, Pennsylvania. Except for periods spent in the experimental apparatus, each duckling was maintained in an individual housing unit consisting of a brown rectangular box $(30 \times 51 \times 30 \mathrm{~cm})$ that was partially filled with bedding material. Under these circumstances, the ducklings could hear each other, but their visual environment was restricted to that provided by the inside of their individual housing units. While in those units, the ducklings had continuous access to food and water.

Apparatus. The experimental apparatus consisted of a plywood box $(120 \times 66 \times 78 \mathrm{~cm})$ divided by a fine-mesh stainless steel screen into two compartments, one for the duckling $(84 \times 66 \times 78 \mathrm{~cm})$ and the other for the imprinting object $(36 \times 66 \times 78 \mathrm{~cm})$. The imprint-
\end{abstract}


ing object consisted of three circular lights (one red, one green, and one blue) mounted vertically on a wood panel that moved horizontally along the length of the stimulus compartment. These lights were each $1.2 \mathrm{~cm}$ in diameter and were spaced some $2.5 \mathrm{~cm}$ apart from each other. Presentations of the moving stimulus were produced by illuminating the colored lights and moving the wood panel back and forth across the stimulus compartment at $5.3 \mathrm{~cm} / \mathrm{sec}$. Presentations of the stationary stimulus were produced by illuminating the colored lights while withholding power from the motor that moved the wood panel. Stimulus absence was accomplished by extinguishing the colored lights and withholding power from the motor.

To permit assessment of the ducklings" locomotor behavior during testing, the carpeted floor of the subject compartment was divided into two unequal portions by a strip of adhesive tape running parallel to, and at a distance of $17 \mathrm{~cm}$ from, the fine-mesh screen of the stimulus compartment. Hence, the resulting small and large areas of the subject compartment were $17 \times 66 \mathrm{~cm}$ and 67 $\times 66 \mathrm{~cm}$, respectively, with the smaller area (the "approach area") nearest the stimulus compartment. A second section of fine-mesh screen (i.e., in addition to the screen that separated the subject and stimulus compartments) was built into one of the side walls of the subject compartment. This screen $(36 \mathrm{~cm}$ high $\times 80 \mathrm{~cm}$ long) permitted the experimenter to observe the exact location of a duckling while it was in the imprinting apparatus.

Lighting in the subject compartment was provided by two continuously illuminated incandescent lamps mounted along the top of the compartment. These lamps were positioned so that unless the imprinting stimulus lights were also illuminated, the light that reflected from the fine-mesh screen prevented the subjects from seeing into the darkened stimulus compartment. The same principle also operated to prevent subjects from seeing the human observer.

Procedure. At 10-16 $\mathrm{h}$ posthatch, each duckling was assigned to either the short-exposure group $(\mathbf{N}=9)$ or the long-exposure group $(\mathrm{N}=9)$, and imprinting sessions were begun. Procedures during these sessions were similar for both groups in that the subjectcompartment lights were dim (7.5-W bulbs were used), the subjects were run singly, and the moving stimulus was presented continuously. Long-exposure subjects, however, received one 30 -min session on each of the first 3 days posthatch, whereas shortexposure subjects received one 10-min session on each of the first 3 days. On the basis of previous research involving similar experimental conditions (Eiserer \& Swope, 1980), it was expected that the 30 total minutes of moving stimulus exposure given to the shortexposure subjects would permit the development of reliable approach to the stimulus should it subsequently be presented as stationary.

A brief preliminary test was given immediately after the third session on Day 3 to verify that both the moving and the stationary stimulus would, in fact, elicit approach from the subjects in both groups. This test, during which the subject compartment remained dimly illuminated, entailed two $60-\mathrm{sec}$ presentations of the moving stimulus and two 60-sec presentations of the stationary stimulus. Across subjects, these two types of stimulus presentation occurred in random order; each stimulus presentation was preceded by a 60 sec period of stimulus absence. In order to ensure that the duckling had to make an active response to accumulate time in the approach area near the imprinting stimulus, the experimenter placed the bird near the center of the subject compartment at the beginning of each of the above $60-\mathrm{sec}$ intervals. Approach was defined as the number of seconds that the duckling spent within the area of the subject compartment near the stimulus compartment, beginning as soon as any portion of the bird's foot touched the strip of tape that bordered the area.

The next phase of the experiment entailed brightening the illumination of the subject compartment, a procedure that is known to increase rates of distress vocalization in 4-day-old ducklings (Eiserer, 1977). On Days 4 and 5 posthatch, two types of approach test were given: dim illumination tests, which involved subjectcompartment lights of $7.5 \mathrm{~W}$ and were identical in procedure to the approach test that occurred on Day 3; and bright illumination tests, which entailed subject-compartment lights of $100 \mathrm{~W}$ but were otherwise identical in procedure to the approach test on Day 3. Each subject in both experimental groups received one dim and one bright illumination test on Day 4 , and then again on Day 5. Order of the tests was counterbalanced across days and across subjects. The intertest interval on a given day was approximately 120 sec.

\section{Results}

In the preliminary test on Day 3 , both groups of ducklings entered the approach area much more during presentations of the moving stimulus (mean = 52 sec per minute for both groups) and the stationary stimulus (mean $=49 \mathrm{sec}$ ) than during stimulus absence $($ mean $=8 \mathrm{sec})[\mathrm{F}(2,32)=223.3$, p $<.01]$. Approach by the long-exposure ducklings was not significantly different from approach by the shortexposure ducklings $[\mathrm{F}(1,16)=0.56, \mathrm{p}>.05]$. And there was no significant interaction between the effects of groups and stimulus condition $[F(2,32)=$ $2.54, \mathrm{p}>.05]$.

The approach tests on Days 4 and 5 (Figure 1) yielded three important effects, as revealed by an analysis of variance (three-factor mixed design: repeated measures on two factors). First, a significant group effect $[F(1,16)=7.39, p<.05]$ indicated that, in general, the long-exposure ducklings approached at higher levels than did the short-exposure duck-

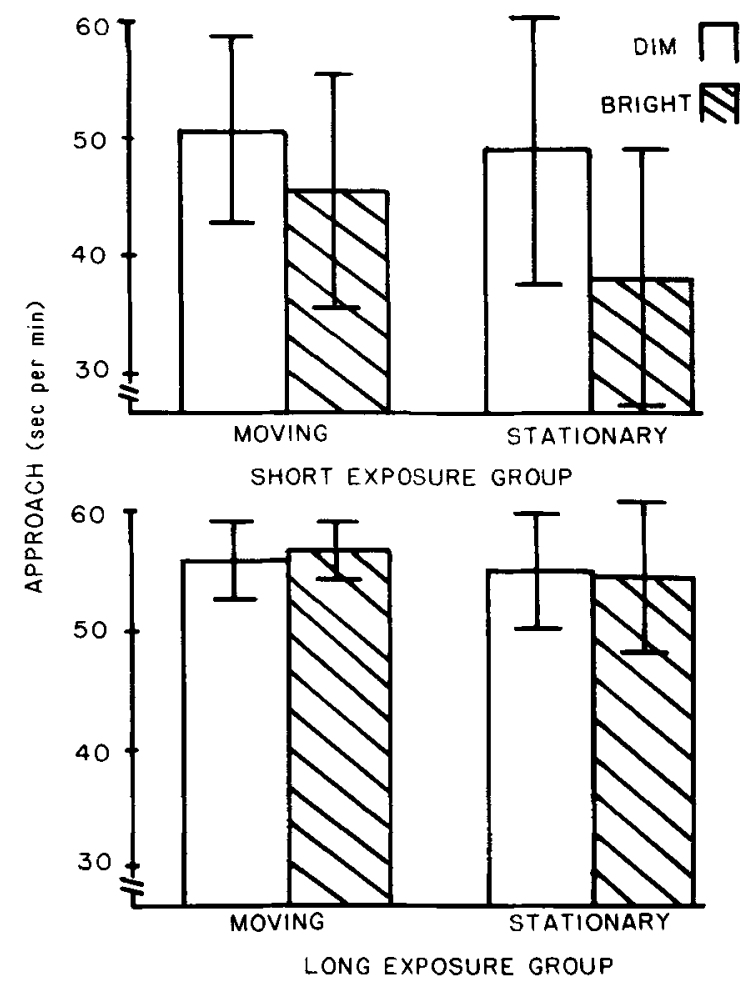

Figure 1. Group mean seconds of approach for short-exposure and long-exposure ducklings during presentations of the moving and stationary object, under both dim and bright illumination in the subject compartment. 
lings. Second, although the overall effect of lighting was significant $[F(1,16)=9.63, p<.05]$, inspection of Figure 1 reveals that the interaction between groups and lighting is more informative; this significant interaction $[F(1,16)=10.49, p<.05]$ indicated that bright illumination led to lowered approach in the short-exposure ducklings but not in the longexposure ducklings. The third finding was a marginally significant interaction between groups and stimulus condition $[F(1,16)=3.46, p<.09]$, which, together with Figure 1, indicated that approach to the stationary stimulus tended to be lower than approach to the moving stimulus in the short-exposure subjects but not in the long-exposure subjects. The other possible effects - the lighting $\times$ stimulus interaction and the groups $\times$ lighting $x$ stimulus interaction-did not approach significance ( $F<1.0$ in both cases).

\section{Discussion}

In the present study, the bright light bulbs themselves represented novel stimuli; additionally, however, the brighter illumination may have indirectly introduced novelty into the environment by making more salient those stimulus aspects of the experimental chamber that had previously been poorly perceived. In any event, the bright illumination did not merely cause perceptual interference (i.e., make the imprinting stimulus harder to see), because, in that case, response deficits also would have occurred in the long-exposure ducklings.

Although the approach test on Day 3 failed to detect a difference in strength of imprinting between the two groups of subjects, such a difference was uncovered in the tests on Days 4 and 5 , when bright illumination was involved. If one accepts that the short-exposure subjects were more weakly imprinted than the long-exposure subjects, then environmental novelty can apparently suppress filial behavior more easily in weakly imprinted subjects than in strongly imprinted ones. This interpretation, of course, is consistent with the conclusions of Sluckin et al. (1979).

Although early studies (Eiserer \& Hoffman, 1973) had suggested that the behavioral control of a stationary imprinting object might be comparable in strength to that of the object in motion, the present results (although of marginal significance) join more recent research in indicating relatively weaker control by a stationary imprinting object (Eiserer, 1977; Gaioni \& Ross, 1982; Sluckin et al., 1979). Thus, the present experiment has essentially replicated the findings of Sluckin et al. despite basic procedural differences in how the stationary object acquired control. This replication, in turn, supports the notion that the two procedures (i.e., seeing the stationary object itself for prolonged periods vs. seeing the stimulus in motion first) are functionally equivalent, so long as the stationary object in the former case is a conspicuous stimulus (Eiserer, 1980).

Approach by the long-exposure birds did not vary under dim versus bright light, but this may have been due more to a "ceiling" problem (the birds approached an average of $55 \mathrm{sec}$ per minute across all conditions) than to ineffectiveness of high illumination per se. Experimenter observations during the tests revealed that the long-exposure ducklings actually followed the imprinting object (as it moved across the stimulus compartment) more vigorously under bright illumination than under dim illumination, even though they remained within the approach area an equivalent amount of time during both conditions. In short, while filial behavior in the shortexposure subjects was disrupted by bright illumination, filial behavior in the long-exposure subjects appeared to be enhanced. Experiment 2 sought to document the enhancement effect by measuring following behavior (rather than simply approach) in longexposure ducklings under varying illumination levels.

\section{EXPERIMENT 2}

\section{Method}

Subjects and Apparatus. Sixteen Khaki Campbell ducklings from two separate hatches were housed under the same conditions as were the subjects in Experiment 1. The experimental apparatus was similar to that described previously, with the addition of two strips of tape that were placed on the carpeted floor parallel to each other and perpendicular to the screen of the stimulus compartment. These strips were located $16 \mathrm{~cm}$ from either side of the subject compartment.

Procedure. Beginning 10-16 h after hatching, each duckling received one 30-min exposure session with the moving stimulus on each of the first 3 days posthatch. The subject-compartment lights during these sessions were dim.

Immediately after the third session on Day 3, the following responses of each duckling were assessed during a 40-min test. During $20 \mathrm{~min}$ of this test, the moving stimulus was presented continuously while the subject-compartment lights were dim; during the other $20 \mathrm{~min}$, the moving stimulus was again presented continuously, but the compartment lights were bright. Half of the subjects were tested under bright lights first, and the other half were tested under dim lights first.

The experimenter monitored the behavior of the duckling throughout the test. A following response was recorded each time any portion of the bird's foot touched or crossed either of the two strips of tape that extended perpendicularly from the screen of the stimulus compartment.

\section{Results}

Observation of the subjects confirmed that virtually all of their responses resulted from attempts to stay as close to the imprinting object as possible, and did not merely result from increases in general activity in the vicinity of the object.

As seen in Figure 2, the number of following responses was greater under bright illumination than under dim illumination. This difference was reliable $[t(15)=2.64, p<.05]$. 


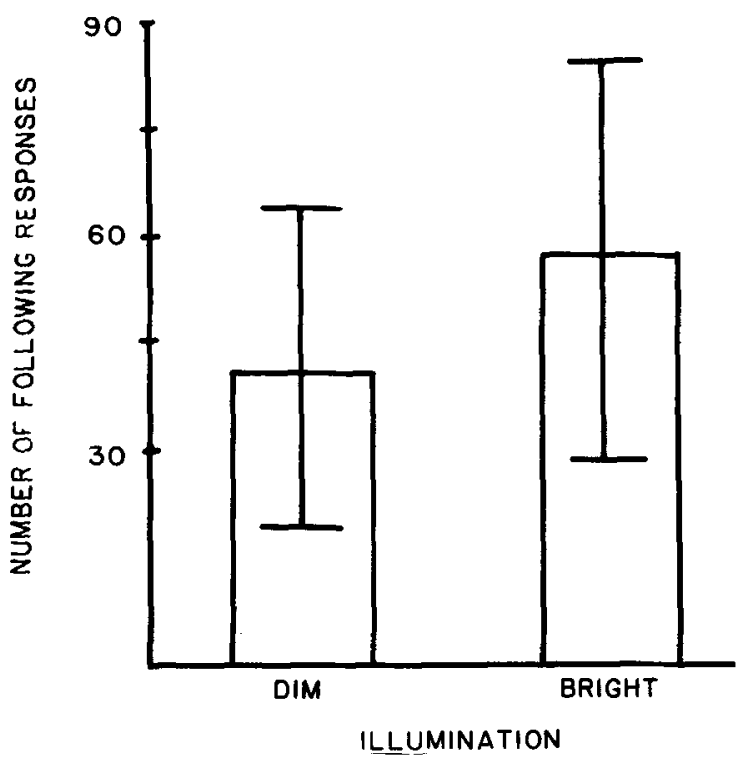

Figure 2. Group mean number of following responses per $20 \mathrm{~min}$ for long-exposure ducklings under both dim and bright illumination.

\section{GENERAL CONCLUSIONS}

The present research has shown that the environmental novelty that accompanies increased illumination can disrupt filial behavior in subjects with little prior imprinting exposure, and yet facilitate filial behavior in subjects who have received lengthy imprinting exposure. In addition, approach to a stationary imprinting object appears to be more easily disrupted than is approach to a moving object.

All of these effects can be understood if one assumes that environmental novelty of the sort generated in the present experiment (1) energizes the dominant (most probable) response in a given situation and (2) elicits responses (immobility or nondirected running) that are incompatible with filial behavior. These two effects (energization and response elicitation) have been postulated by authors in other contexts (most notably, Amsel, 1958, regarding anticipatory frustration).

In weakly imprinted ducklings, competing responses elicited by environmental novelty might be dominant over filial approach tendencies, and would thus disrupt the latter. Since a stationary imprinting object is presumed to be less powerful in eliciting filial responses than is a moving object, approach to the stationary object should be more vulnerable to competing responses. Finally, in strongly imprinted ducklings exposed to the moving object, filial ten- dencies could prevail over competing responses and hence benefit from the energization effects of environmental novelty.

\section{REFERENCES}

Amse L, A. The role of frustrative nonreward in noncontinuous reward situations. Psychological Bulletin, 1958, 55, 102-119.

Brown, R. T., \& Hamilton, A. S. Imprinting: Effects of discrepancy from rearing conditions on approach to a familiar imprinting object in a novel situation. Journal of Comparative and Physiological Psychology, 1977, 91, 784-793.

Candland, D. K., Nagy, Z. M., \& Conkeyn, D. H. Emotional behavior in the domestic chicken as a function of age and developmental environment. Journal of Comparative and Physiological Psychology, 1963, 56, 1069-1073.

Eiserer, L. A. Behavioral control by stimulus components of an imprinting object. Animal Learning \& Behavior, 1977, 5, 153-160.

Eiserer, L. A. Anomalous effects in psychology: Parallels between the brain stimulation and imprinting literatures. Psychological Record, 1978, 28, 557-570.

Erserer, L. A. Development of filial attachment to static visual features of an imprinting object. Animal Learning \& Behavior, $1980,8,159-166$.

Eise rer, L. A., \& Horfman, H. S. Priming of ducklings' responses by presenting an imprinted stimulus. Journal of Comparative and Physiological Psychology, 1973, 82, 345-359.

Eiserer, L. A., \& Swope, R. L. Acquisition of behavioral control by static visual features of an imprinting object. Animal Learning \& Behavior, 1980, 8, 481-484.

Fischer, G. J. Arousal and impairment: Temperature effects on following during imprinting. Journal of Comparative and Physiological Psychology, 1970, 73, 412-420.

Gaioni, S. J., \& Ross, L. E. Distress calling induced by reductions in group size in ducklings reared with conspecifics or imprinting stimuli. Animal Learning \& Behavior, 1982, 10, 521-529.

Hess, E. H. Imprinting. Science, 1959, 130, 133-141.

Hoffman, H. S., Eiseren, L. A., \& Singer, D. Acquisition of behavioral control by a stationary imprinting stimulus. Psychonomic Science, 1972, 26, 146-148.

MolTz, H. Imprinting: Empirical basis and theoretical significance. Psychological Bulletin, 1960, 57, 291-314.

Moltz, H., Rosenblum, L., \& Halikas, N. Imprinting and level of anxiety. Journal of Comparative and Physiological Psychology, 1959, 52, 240-244.

Pitz, G. F., \& Ross, R. B. Imprinting as a function of arousal. Journal of Comparative and Physiological Psychology, 1961, $54,602-604$.

SAlzeN, E. A. Imprinting in birds and primates. Behaviour, 1967, 28, 232-254.

SAlzen, E. A. Imprinting and environmental learning. In L. R. Aronson, E. Tobach, D. S. Lehrman, \& J. S. Rosenblatt (Eds.), Development and evolution of behavior. San Francisco: Freeman, 1970.

Sluckin, W. Imprinting and early learning. London: Methuen, 1972.

Sluckin, W., Berryman, J. C., Mayes, A., \& Mann, D. Chicks' responses to familiar stimuli in unfamiliar environments. Quarterly Journal of Experimental Psychology, 1979, 31 , 701-710.

(Manuscript received February 1, 1983; revision accepted for publication June 10, 1983.) 